BASIC RESEARCH

\title{
Quantitative assessment of regional peak myocardial acceleration during isovolumic contraction and relaxation times by tissue Doppler imaging
}

\author{
I Hashimoto, X-K Li, A Hejmadi Bhat, M Jones, D J Sahn
}

Heart 2005;91:81 1-816. doi: 10.1136/hrt.2004.033845

See end of article for authors' affiliations

.....................

Correspondence to: Dr David J Sahn, L608, Pediatric Cardiology, Oregon Health \& Science University, 3181 SW Sam Jackson Park Road,

Portland, Oregon 972393098, USA; sahnd@ohsu. edu

Accepted 18 August 2004
Objective: To examine regional wall acceleration and its relation to relaxation.

Study design: 8 sheep were examined by tissue Doppler ultrasound imaging (VingMed Vivid FiVe) in apical four chamber views to evaluate the left ventricular wall divided into six segments and the mitral annulus in two segments. Peak myocardial acceleration during isovolumic periods (pIVA) derived from tissue Doppler echocardiography was analysed during isovolumic contraction (ICT) and relaxation times (IRT) in each segment.

Interventions: After scanning at baseline, haemodynamic status was changed by administration of blood, dobutamine, and metoprolol. Changes of pIVA during IRT and ICT were compared over the four haemodynamic conditions in parallel with their peak positive and negative $\mathrm{dP} / \mathrm{dt}$ measured with a high frequency manometer tipped catheter.

Results: pIVA of the basal lateral segment during ICT correlated most strongly with peak positive $\mathrm{dP} / \mathrm{dt}$ $(r=0.96, p<0.0001)$ and there was good correlation between pIVA of the mitral valve annulus in the septum during IRT and peak negative $\mathrm{dP} / \mathrm{dt}(r=0.80, \mathrm{p}<0.0001)$. pIVA differed significantly between the four haemodynamic conditions during ICT in all segments ( $p<0.05$ ); pIVA during IRT did not differ significantly between the four conditions.

Conclusions: pIVA of the basal lateral wall during ICT correlated most strongly with peak positive $\mathrm{dP} / \mathrm{dt}$, and pIVA of the septal mitral valve annulus during IRT correlated well with peak negative $\mathrm{dP} / \mathrm{dt}$.
$\mathrm{T}$ issue Doppler imaging (TDI) provides a method for assessing regional wall velocity with a high spatial and temporal resolution on a two dimensional colour map. ${ }^{1-3}$ TDI can be used to asses not only regional wall abnormalities but also global ventricular function. The tissue velocity of the myocardium is smaller than that of blood flow but ventricular wall motion accelerates considerably because activation of cardiac muscle cells induces muscle contraction, generating tissue deformation and wall motion. Strain rate imaging has been developed to analyse such tissue deformation and has been reported to be useful for evaluating global left ventricular (LV) function..$^{4-6}$

TDI acceleration is a derivative measurement obtained from tissue velocity. High temporal and spatial resolution makes it possible to display and detect intramural abnormal conduction in the heart. This isovolumic deflection has been used to detect the pre-excitation region in patient with the Wolff-Parkinson-White syndrome. ${ }^{78}$ Myocardial acceleration and ventricular cavity pressure change rate $(\mathrm{dP} / \mathrm{dt})$, which has been used as an index of contractility or relaxation, are altered in parallel because intracavity pressure change is caused by myocardial contraction or relaxation, especially during their respective isovolumic periods. Vogel and colleagues $^{9}$ reported that myocardial acceleration during isovolumic contraction time (ICT) was a good index of contractility, since it was not particularly preload or afterload dependent. ${ }^{9}$

On the other hand, longitudinal myocardial relaxation has also been reported to have a relation to LV diastolic function independent of the preload condition. ${ }^{10-12}$

The purposes of the present study were to evaluate the feasibility of using peak myocardial acceleration during isovolumic periods (pIVA) as an index of LV contractility and relaxation; and to determine the most appropriate ventricular wall segment for evaluating LV function by pIVA.

\section{METHODS}

\section{Experimental preparation}

Eight sheep weighing 35-47 kg (mean (SD) 40.1 (4.2) kg) were studied. All sheep underwent thoracotomy under general anaesthesia induced with intravenous sodium pentobarbital (25 mg/kg body weight) and maintained with $1-2 \%$ isoflurane with oxygen. The sheep were intubated and ventilated with a volume-cycle respirator. An ECG was monitored from limb leads. Intracavity manometer tipped catheters (model SPC-350, Millar Instruments, Inc, Houston, Texas, USA) were placed in the LV through a carotid artery and in the left atrium through the appendage for pressure recording. Positive and negative peak $\mathrm{dP} / \mathrm{dt}$ were obtained from derivatives of the pressure curve of the LV. Positive peak $\mathrm{dP} / \mathrm{dt}$ was used to evaluate global systolic function and negative peak $\mathrm{dP} / \mathrm{dt}$ was used to evaluate global diastolic function. ${ }^{6}{ }^{13-15}$ Another catheter was positioned in the femoral artery to monitor systemic arterial pressure and blood gas. These catheters were interfaced with a physiological recorder (ES 2000, Gould Inc) with a fluid filled pressure transducer (model PD231D, Gould Statham). Two electromagnetic flow probes (model EP455, Carolina Medical Electronics, Inc, King, North Carolina, USA) were placed to measure cardiac output: one around the skeletonised ascending aorta distal to

Abbreviations: AL, apical lateral; AS, apical septal; BL, basal lateral; $B S$, basal septal; ICT, isovolumic contraction time; IRT, isovolumic relaxation time; LV, left ventricular; ML, mid-lateral; MS, mid-septal; $M V L$, lateral mitral valve annulus; MVS, septal mitral valve annulus; pIVA, peak myocardial acceleration during isovolumic periods; TDI, tissue Doppler imaging 
the coronary ostia and proximal to the bronchiocephalic trunk, and the second around the pulmonary artery just above the pulmonary valve. Both flow probes were connected to flowmeters (model FM501, Carolina Medical Electronics) and interfaced with the same physiological recorder (ES 2000) that was used for pressure recording. All haemodynamic data were recorded at paper speed of $250 \mathrm{~mm} / \mathrm{s}$. Four consecutive cardiac cycles were analysed for each haemodynamic determination. All operative and animal management procedures were approved by the Animal Care and Use Committee of the National Heart, Lung, and Blood Institute.

\section{Experimental protocol}

Baseline, volume loading, dobutamine infusion, and metoprolol infusion were used to produce four different haemodynamic conditions for each animal. After a baseline recording, $500 \mathrm{ml}$ of blood was infused slowly, then intravenous dobutamine $(2-10 \mu \mathrm{g} / \mathrm{kg} / \mathrm{min})$ and $5 \mathrm{mg}$ of metoprolol were administered at least one hour apart from the previous stage. We always started the next stage after physiological status returned to the baseline condition. All of the haemodynamic and myocardial velocity data (described below) were acquired simultaneously at each haemodynamic stage with a short period of suspended ventilation for the duration of data acquisition.

\section{Echocardiographic analysis}

We used a Vivid FiVe digital ultrasound system (GE/VingMed Ultrasound, Horten, Norway) for the present study. Scans were recorded longitudinally from the apex to acquire an apical four chamber view with a $5.0 \mathrm{MHz}$ phased array transducer. TDI data were acquired with a pulse repetition frequency from $1.0-4.5 \mathrm{kHz}$ and a frame rate varying from 80-130 frames/s maximised as possible. The TDI sector angle was limited to that required to encompass the LV cavity and walls, and line density and packet size were adjusted for smooth consistent data with maximised frame rate. With these settings no aliasing of velocities was encountered. TDI data for the two dimensional images for two to three cycles for each stage were stored on magnetic optical disk for each stage, with subsequent offline analysis of scan line based digital data. Optimised TDI data for the two dimensional images of the heart for each stage and amplitude were analysed by the EchoPac 6.3 archiving application software of the Vivid FiVe. This software allowed us to evaluate several locations of tissue velocity simultaneously and to display velocity-time relation curves for each.

According to the generally accepted standardised myocardial segmentation, we divided the LV wall into six segments: basal lateral (BL), mid-lateral (ML), apical lateral (AL), basal septal (BS), mid-septal (MS), and apical septal (AS). We then measured the velocity of each segment shown in fig $1 \mathrm{Al}$, Bl. ${ }^{16}$ In addition, we measured velocities of the septal (MVS) and lateral mitral valve annulus (MVL). Eight sampling points in total on the LV wall were measured in the present study. To keep the same volume of muscle in each sampling region, the autotracking technique was used for all measurements. Autotracking for each sampling point was carefully
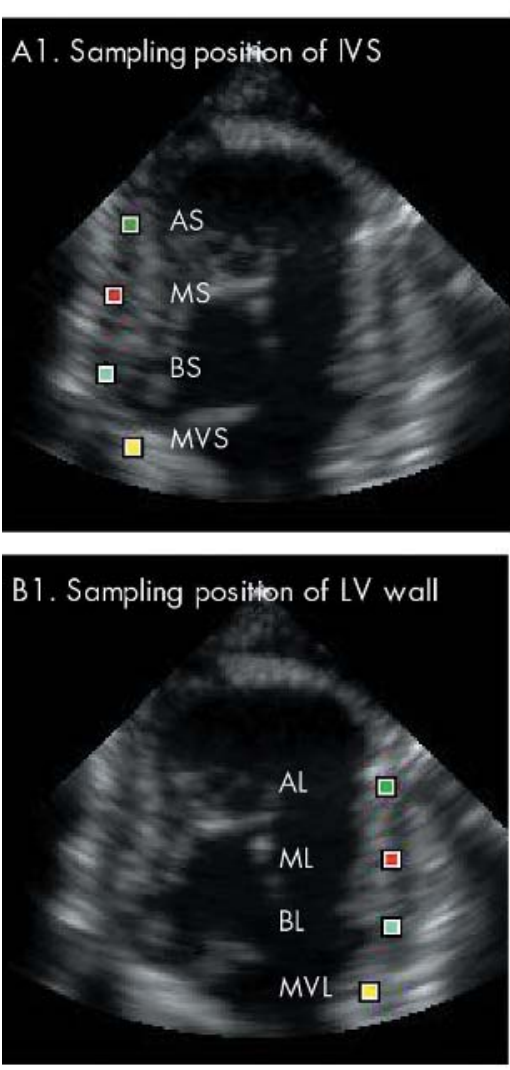
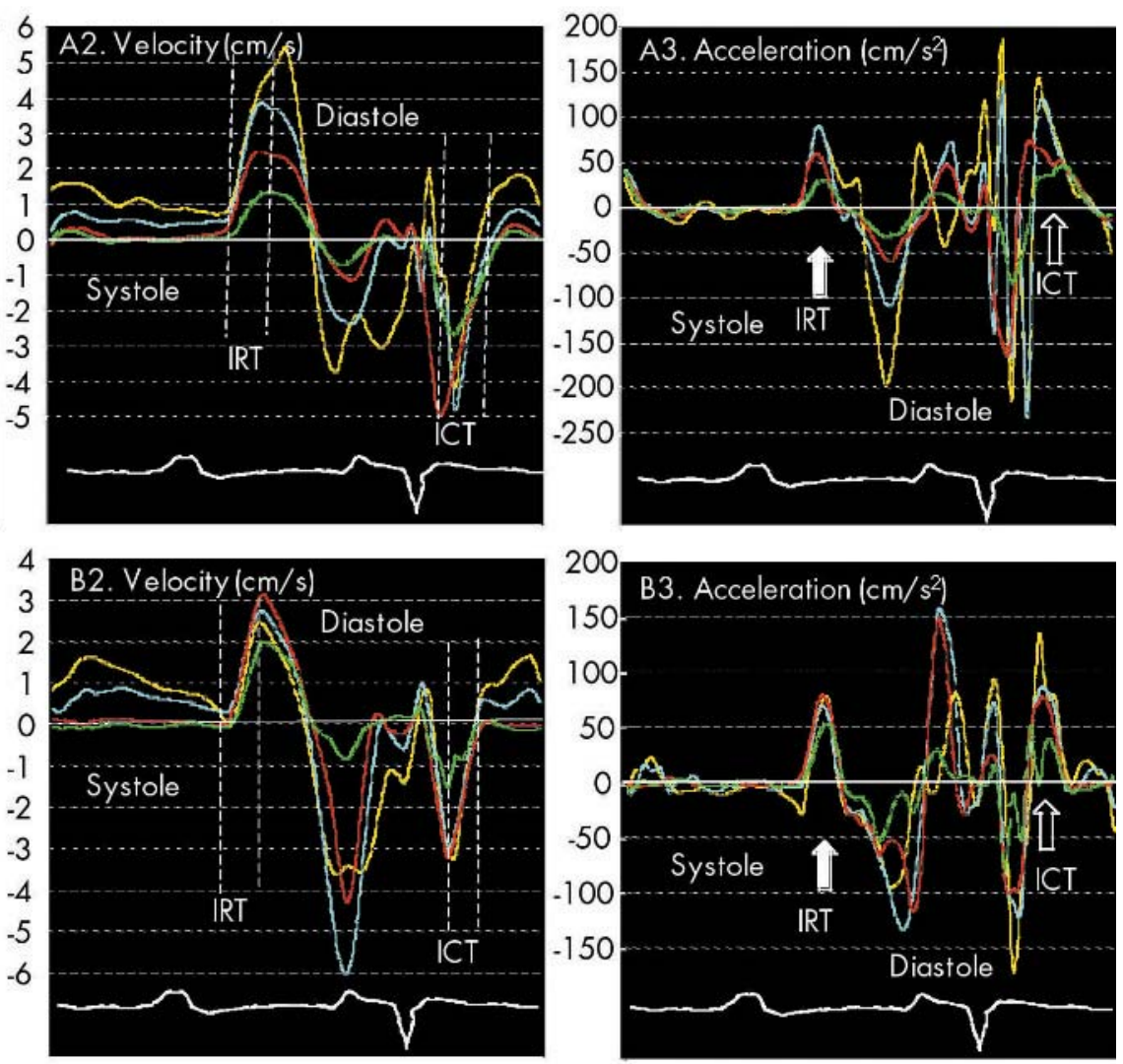

Figure 1 Tissue Doppler imaging (TDI) sampling positions for the interventricular septum (IVS) and left ventricular (LV) lateral wall. The LV wall was divided into six wall and two annular segments according to American Heart Association standardised myocardial segmentation. IVS (A1), velocity (A2), and acceleration curves (A3) are shown, each segment trace matching the colour of the sampling position: mitral valve annulus septal (MVS, yellow), basal septal (BS, blue), mid septal (MS, red), apical septal (AS, green). The same positions on the lateral wall (B1) match the velocity (B2) and acceleration curves (B3) for lateral mitral valve annulus (MVL, yellow), basal lateral wall (BL, blue), mid lateral wall (ML, red), and apical lateral wall (AL, green). Isovolumic relaxation time (IRT) and isovolumic contraction time (ICT) are shown for each velocity (A2, B2) and acceleration curve (A3, B3). Definite positive peaks on acceleration curves were noted during IRT (solid white arrow) and ICT (outlined black arrow). 
Table 1 Haemodynamic parameters in each condition

\begin{tabular}{lllll}
\hline & Baseline & Blood loading & Dobutamine & Metoprolol \\
\hline Cardiac output $(\mathrm{l} / \mathrm{min})$ & $1.62(0.47)$ & $2.2(0.48)^{*}$ & $2.0(0.48)$ & $1.54(0.24)$ \\
Heart rate (beats/min) & $100(12)$ & $106(6)$ & $142(25)^{*}$ & $81(8)^{*}$ \\
LVEDP $(\mathrm{mm} \mathrm{Hg})$ & $12.1(4.2)$ & $16.2(6.2)$ & $11.1(5.9)$ & $15.3(2.1)$ \\
LAP $(\mathrm{mm} \mathrm{Hg})$ & $8.9(3.0)$ & $13.5(4.0)^{* *}$ & $9.1(2.5)^{*}$ & $10.7(2.9)$ \\
Peak dP/dt $(\mathrm{mm} \mathrm{Hg} / \mathrm{s})$ & $1110(324)$ & $1697(299)^{*}$ & $2260(558)^{*}$ & $735(250)^{*}$ \\
Peak $-\mathrm{dP} / \mathrm{dt}(\mathrm{mm} \mathrm{Hg} / \mathrm{s})$ & $-1083(183)$ & $-1290(137)^{*}$ & $-1391(295)^{*}$ & $-667(277)^{*}$ \\
\hline
\end{tabular}

${ }^{*} p<0.05$ compared with baseline; ${ }^{* *} p<0.005$ compared with baseline.

LAP, left atrial pressure; LVEDP, left ventricular end diastolic pressure.

set not to deviate from the wall zone. Autotracking is a newly developed modality that allows semiautomatic correction of the sampling position tracking specific tissue speckles throughout heart cycle.

After frame by frame velocity values were obtained, myocardial velocity acceleration was calculated as the difference between two sequential velocities divided by the frame by frame time interval. The time interval for calculation was consistently set from $20-25 \mathrm{~ms}$ averaging two to three consecutive velocity values. Acceleration curves were obtained from each calculation and displayed as in fig lA3 and fig 1B3. Acceleration curves had positive peaks during isovolumic relaxation time (IRT) and ICT, respectively. ICT was determined by the Q wave on the ECG and by closing of the mitral valve on the two dimensional echocardiographic cine loop. IRT was also determined by the opening of the mitral valve on the cine loop. We analysed pIVAs during both IRT and ICT in each wall segment and mitral valve annulus.

\section{Statistical analysis}

All data were expressed as mean (SD). One way repeated measures analysis of variance was performed to compare pIVAs between the four haemodynamic conditions, and Dunnett's test was used for post hoc analysis. Linear regression analysis was used for comparison between pIVA values and peak $\mathrm{dP} / \mathrm{dt}$ as a haemodynamic parameter. All statistical analyses were performed with StatView 5.01 (SAS Institute, Cary, North Carolina, USA). A probability value of $\mathrm{p}<0.05$ was regarded as significant.

\section{RESULTS}

Table 1 shows the haemodynamic data related to each stage. Cardiac output significantly increased from 1.62 (0.47) l/min to $2.2(0.48) \mathrm{l} / \mathrm{min}$ with blood loading and to $2.0(0.48) \mathrm{l} / \mathrm{min}$ with dobutamine infusion. Heart rate also significantly changed from 100 (12) beats/min to 142 (25) beats/min with dobutamine infusion. LV end diastolic pressure did change significantly between stages. But left atrial pressure changed significantly from baseline with blood loading $(\mathrm{p}<0.005)$ and with dobutamine infusion $(\mathrm{p}<0.05)$. Both positive peak $\mathrm{dP} / \mathrm{dt}$ and negative peak $\mathrm{dP} / \mathrm{dt}$ also changed significantly $(\mathrm{p}<0.05)$.
Figure 1 shows the sampling positions (fig $1 \mathrm{Al}, \mathrm{Bl}$ ), velocities (fig 1A2, B2), and acceleration curves (fig 1A3, B3). In fig $1 \mathrm{~A}$, yellow, blue, red, and green lines show velocity and acceleration of MVS, BS, MS, and AS, respectively. In fig 1B, yellow, blue, red, and green lines show velocity and acceleration of MVL, BL, ML, and AL, respectively. In systole, positive wall motion of each segment was noted, corresponding to myocardial contraction, and the velocity of each segment gradually decreased from the mitral valve annulus to the apex along both the IVS and LV wall. However, no myocardial acceleration was observed during systole. On the other hand, in diastole, persistent negative acceleration of myocardium was observed in each segment, corresponding to early and late diastolic velocity waves. Also, during IRT and ICT, acceleration curves had definite positive peaks at all sampling points (fig 1A3, B3). Myocardial acceleration maps confirmed that the myocardium accelerated in the IVS and LV wall during both IRT and ICT (fig 2A, B).

Figure 3 shows a representative velocity and corresponding acceleration curves obtained from the BS at each haemodynamic condition. Figure 3A shows the baseline data. Definite positive peaks were observed during both IRT and ICT in the acceleration curves (dVel/dt). pIVA during ICT was increased by blood loading (fig 3B) and its amplitude was significantly augmented by dobutamine infusion (fig 3C). However, no myocardial acceleration was observed during systole. In contrast, metoprolol infusion significantly decreased pIVA amplitude (fig 3D). However, pIVA during IRT was decreased by metoprolol infusion but was not significantly augmented by blood and dobutamine infusion.

Table 2 shows the changes of pIVA for each segment during IRT produced by each haemodynamic condition. Only pIVA of ML and AL were significantly altered; the other segments did not change significantly with each haemodynamic condition. On the other hand, pIVA during ICT differed significantly between the four haemodynamic conditions and pIVA increased significantly, especially with dobutamine infusion over all ventricular segments (table 3 ). Blood loading increased pIVA during ICT but this change was not significant in the IVS. The pIVA during ICT of MVL and ML increased significantly with blood loading (fig 4).
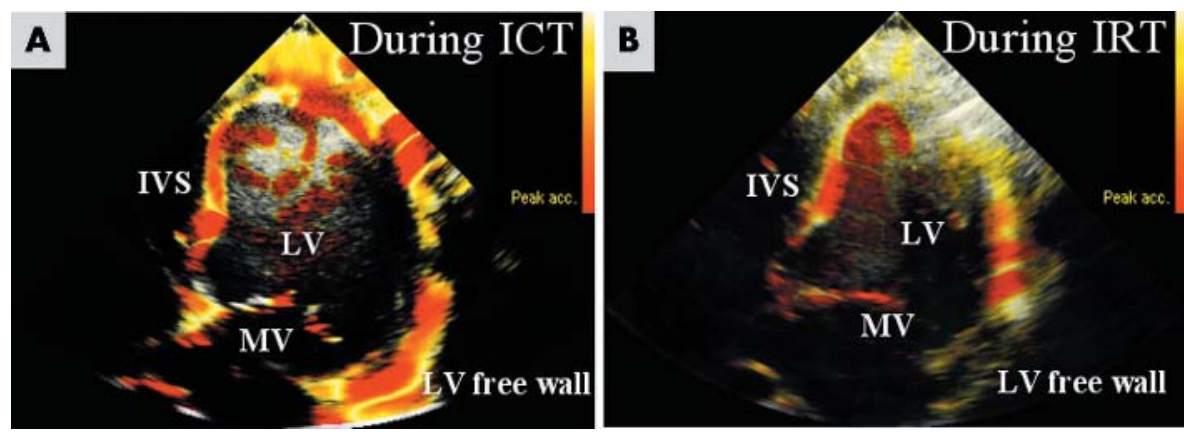

Figure 2 Myocardial acceleration map during (A) ICT and (B) IRT. Myocardial segments that accelerated during ICT or IRT appear red. MV, mitral valve. 

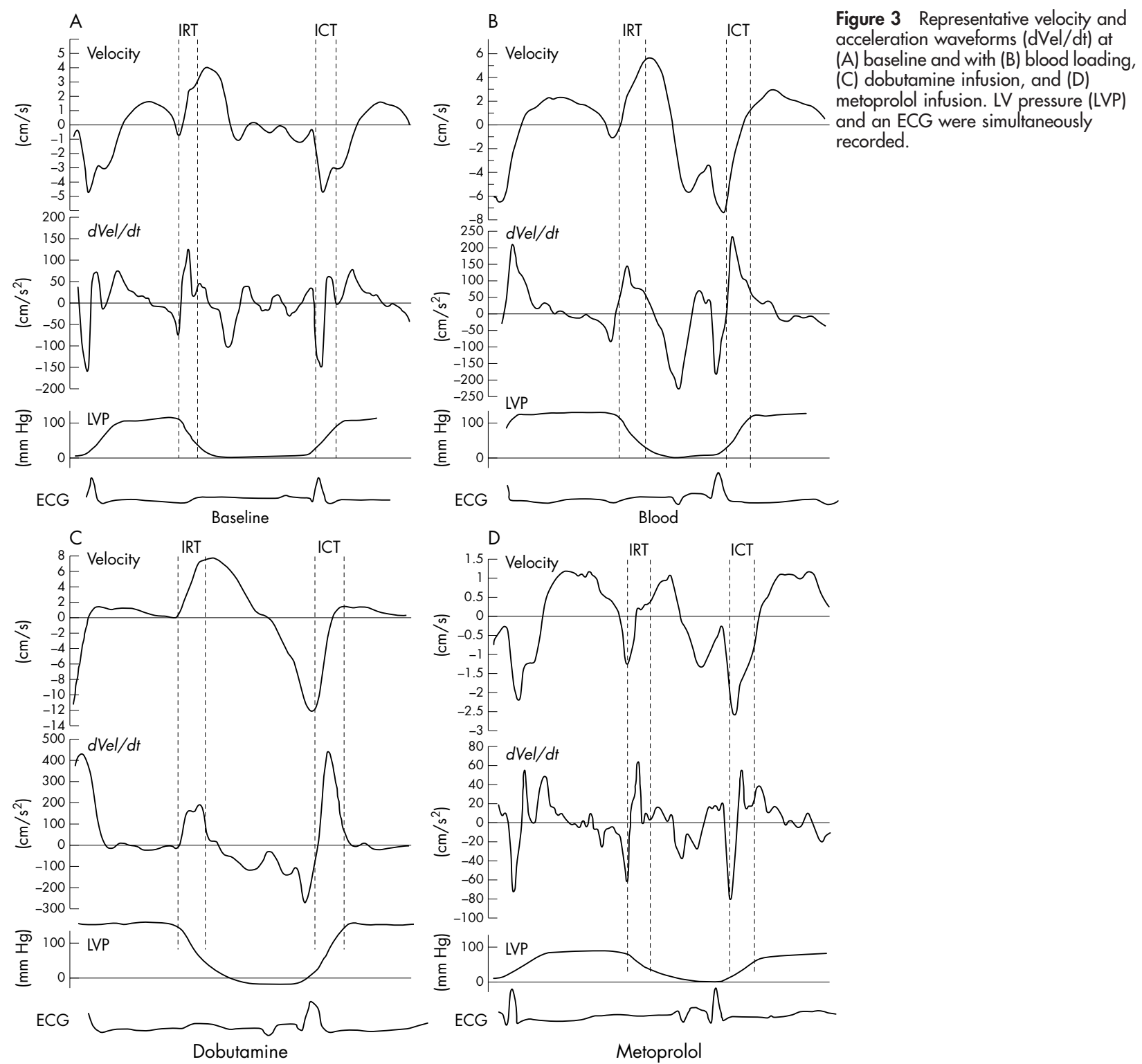

Significant differences of pIVA between each segment were observed during IRT as well as ICT (table 2, table 3).

Table 4 shows the correlations between peak negative or positive $\mathrm{dP} / \mathrm{dt}$ and pIVA during IRT or ICT in each LV wall segment. There was a significant correlation between peak positive $\mathrm{dP} / \mathrm{dt}$ and pIVA during ICT in all segments. BL correlated most strongly with them $(r=0.96, \mathrm{p}<0.0001)$ (fig 4). On the other hand, correlation between negative $\mathrm{dP} /$ $\mathrm{dt}$ and pIVA during IRT was weak in the LV lateral wall. In the IVS, especially in the MVS, there was a good correlation between pIVA during IRT and peak negative $\mathrm{dP} / \mathrm{dt}(r=0.80$, $\mathrm{p}<0.0001$ ) (fig 4).

Table 2 Haemodynamic response of peak myocardial acceleration $\left(\mathrm{cm} / \mathrm{s}^{2}\right)$ during isovolumic relaxation time (IRT) for each wall segment

\begin{tabular}{llllll}
\hline Segment & Baseline & Blood loading & Dobutamine & Metoprolol & p Value \\
\hline MVL & $87.0(47.3)$ & $93.2(38.0)$ & $100.1(0.48)$ & $59.9(30.3)$ & 0.18 \\
BL & $95.2(39.2)$ & $100.9(54.3)$ & $111.7(44.9)$ & $74.2(45.9)$ & 0.38 \\
ML & $72.0(30.1)$ & $94.6(30.1)$ & $79.7(30.3)$ & $54.1(29.0)$ & 0.044 \\
AL & $83.7(29.4)$ & $77.9(31.0)$ & $92.3(46.9)$ & $39.0(19.9)$ & 0.041 \\
MVS & $109.9(33.2)$ & $106.2(30.9)$ & $143.1(65.6)$ & $70.2(29.8)$ & 0.30 \\
BS & $70.1(28.2)$ & $92.0(35.3)$ & $93.6(55.1)$ & $56.3(26.0)$ & 0.22 \\
MS & $51.8(25.7)$ & $73.8(22.0)$ & $71.4(25.8)$ & $60.5(44.8)$ & 0.27 \\
AS & $45.6(24.6)$ & $42.7(25.8)$ & $48.6(35.5)$ & $48.6(35.5)$ & 0.37 \\
\hline
\end{tabular}

AL, apical lateral; $A S$, apical septal; BL, basal lateral; BS, basal septal; ML, mid-lateral; MS, mid-septal; MVL, lateral mitral valve annulus; MVS, septal mitral valve annulus. 
Table 3 Haemodynamic response of peak myocardial acceleration $\left(\mathrm{cm} / \mathrm{s}^{2}\right)$ during isovolumic contraction time (ICT) for each wall segment

\begin{tabular}{llllll}
\hline Segment & Baseline & Blood loading & Dobutamine & Metoprolol & p Value \\
\hline MVL & $146.3(69.7)$ & $249.7(137.1)^{* *}$ & $332.9(131.2)^{* *}$ & $89.2(42.3)$ & 0.0005 \\
$\mathrm{BL}$ & $164.5(30.9)$ & $256.3(58.9)$ & $277.1(126.0)^{*}$ & $126.6(52.3)$ & 0.012 \\
$\mathrm{ML}$ & $142.7(40.2)$ & $247.4(93.3)^{*}$ & $278.6(76.7)^{*}$ & $84.4(20.5)^{*}$ & 0.0002 \\
$\mathrm{AL}$ & $131.7(35.9)$ & $202.9(35.9)$ & $288.8(91.4)^{*}$ & $66.3(29.0)^{*}$ & 0.0001 \\
$\mathrm{MVS}$ & $159.6(62.9)$ & $263.8(89.3)$ & $305.5(126.5)^{*}$ & $96.2(48.9)$ & 0.010 \\
$\mathrm{BS}$ & $175.5(81.5)$ & $240.3(61.4)$ & $298.4(131.2)^{*}$ & $83.0(31.3)^{*}$ & 0.0007 \\
$\mathrm{MS}$ & $132.6(78.9)$ & $173.2(92.2)$ & $276.3(109.8)$ & $78.9(58.1)^{*}$ & 0.033 \\
$\mathrm{AS}$ & $101.6(46.2)$ & $96.7(42.0)$ & $191.1(88.2)^{*}$ & $67.7(21.4)$ & 0.036 \\
\hline \multirow{2}{*}{$\mathrm{p}<0.05$ compared with baseline; ${ }^{* *} \mathrm{p}<0.0005$ compared with baseline. } & &
\end{tabular}

\section{DISCUSSION}

In the present study, we showed the feasibility of using pIVA for evaluating LV contractility and relaxation along with regional analysis of ventricular wall function. TDI is a newly developed modality that allows us to measure the velocity of the ventricular wall with high spatial and temporal resolutions. Myocardial acceleration can be obtained from the derivative of the velocity curve and allows detection of the pre-excitation region of the Wolff-Parkinson-White syndrome or other abnormalities in conduction. ${ }^{78}$

We calculated myocardial acceleration as the difference between two sequential velocities divided by the frame by frame time interval. The time interval for calculation, which was determined by the frame rate, was consistently set from 20-25 ms, averaging two to three consecutive velocity values because raw velocity data obtained frame by frame still contained noise components and had the possibility of leading to miscalculation. Therefore, averaging of consecutive velocity values may eliminate the noise but may lose velocity information during isovolumic periods. A high frame rate is considered to be an important instrumental preset for evaluating pIVA. We used a rate of about $100 \mathrm{frames} / \mathrm{s}$ in almost every experiment and averaged two or three consecutive velocities to display the acceleration curve. There were no experimental settings in which we could not identify both pIVAs during IRT and ICT in the present study.

Autotracking was a useful application to keep the same sampling region and enabled the segmental wall analysis in the present study. In conventional sampling, it was impossible to measure the velocity of a specific wall segment continuously because the wall moves but the sampling position is fixed. We applied this tracing technique to all measurements in this study.
Our data showed that tissue Doppler derived pIVA correlated differently with peak $\mathrm{dP} / \mathrm{dt}$ depending on the ventricular segment. Average amplitudes of pIVA were also significantly different depending on the ventricular segment. pIVA amplitudes were maximal in the mitral valve annulus and BS or BL wall segments, and pIVA gradually decreased from base to apex similarly to velocity difference. ${ }^{17}{ }^{18}$ During ICT, there was a good correlation between pIVA and peak positive $\mathrm{dP} / \mathrm{dt}$ in both the IVS and the LV lateral wall. In the present study, pIVA of BL correlated most strongly with peak positive $\mathrm{dP} / \mathrm{dt}$. Although the amplitude of pIVA of the AL or AS segment was significantly smaller than that of the BL or BS segments, there was a good correlation between them in both the IVS and LV wall. Conversely, although there was a good correlation between pIVA of the IVS during IRT and negative $\mathrm{dP} / \mathrm{dt}$, pIVA of the LV wall during IRT did not correlate well with negative $\mathrm{dP} / \mathrm{dt}$. Unlike contraction, relaxation is a passive ventricular event caused by early diastolic filling and atrial contraction and may occur longitudinally to the heart. ${ }^{10}{ }^{12} 18$ When scanned longitudinally from the apex, while the IVS moves longitudinally along the ultrasound, the LV free wall moves in both lateral and longitudinal directions. This may make it hard to interpret the velocity vector of the LV lateral wall especially during IRT.

In the clinical situation, a preload independent marker is needed to evaluate ventricular function. Pressure derived dP/ $\mathrm{dt}$ is well known to indicate a significant load dependence compared with a conductance catheter derived index (for example, the end systolic pressure-volume relation). ${ }^{19}$ Ultrasound derived strain rate has been reported to be a load dependent marker.5 In the present study, ultrasound derived pIVA was relatively insensitive to blood loading but was sensitive to an inotropic agent during ICT. Our data are
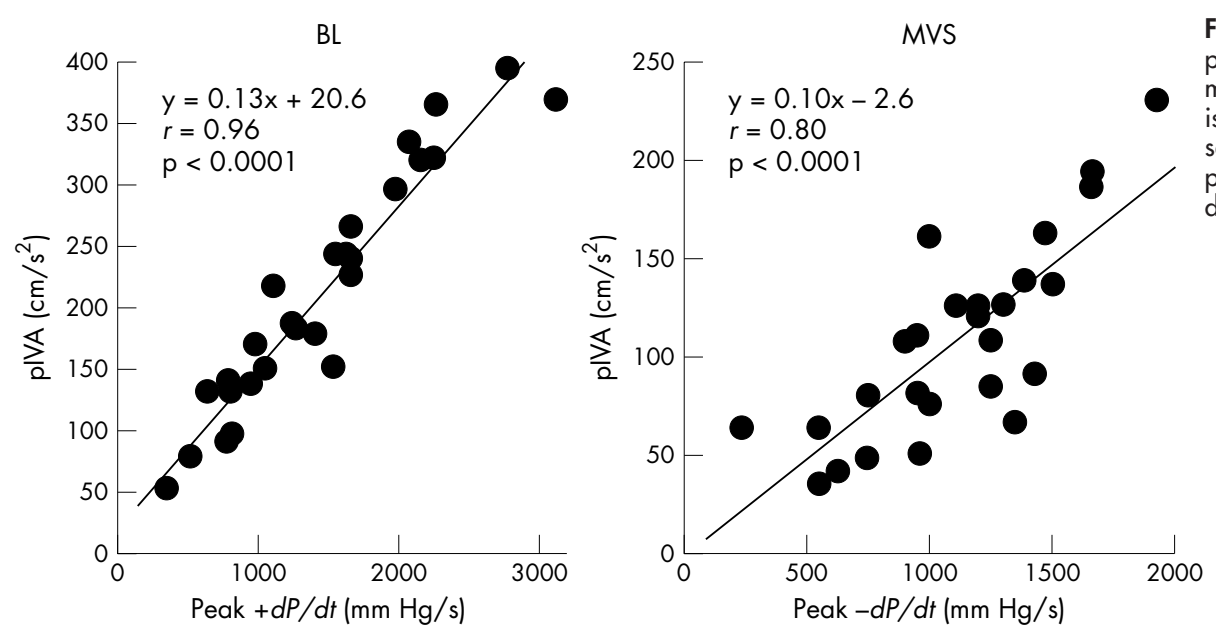

Figure 4 Correlations between (left) peak positive $\mathrm{dP} / \mathrm{dt}$ and peak myocardial acceleration during isovolumic periods ( $\mathrm{plVA}$ ) of the BL segment during ICT and (right) absolute peak negative $\mathrm{dP} / \mathrm{dt}$ and $\mathrm{pIVA}$ of MVS during IRT. 
Table 4 Correlations between peak negative or positive $\mathrm{dP} / \mathrm{dt}$ and peak myocardial acceleration during IRT or ICT in each wall segment

\begin{tabular}{|c|c|c|c|c|}
\hline \multirow[b]{2}{*}{ Segment } & \multicolumn{2}{|c|}{$\begin{array}{l}\text { Peak }-\mathrm{dP} / \mathrm{dt} \text { and pIVA } \\
\text { during IRT }\end{array}$} & \multicolumn{2}{|c|}{$\begin{array}{l}\text { Peak dP/dt and plVA during } \\
\text { ICT }\end{array}$} \\
\hline & $\mathrm{CC}$ & p Value & $\mathrm{CC}$ & p Value \\
\hline MVL & 0.54 & 0.0024 & 0.82 & $<0.0001$ \\
\hline $\mathrm{BL}$ & 0.29 & 0.132 & 0.96 & $<0.0001$ \\
\hline$M L$ & 0.56 & 0.0016 & 0.87 & $<0.0001$ \\
\hline $\mathrm{AL}$ & 0.63 & 0.0003 & 0.89 & $<0.0001$ \\
\hline MVS & 0.80 & $<0.0001$ & 0.83 & $<0.0001$ \\
\hline BS & 0.73 & $<0.0001$ & 0.85 & $<0.0001$ \\
\hline MS & 0.75 & $<0.0001$ & 0.74 & $<0.0001$ \\
\hline AS & 0.60 & 0.0007 & 0.52 & 0.0042 \\
\hline
\end{tabular}

${ }^{*} p<0.05$ compared with baseline; ${ }^{* *} p<0.0005$ compared with baseline. CC, correlation coefficient; pIVA, peak myocardial acceleration during isovolumic periods.

in agreement with the experience of Vogel and colleagues. ${ }^{9}$ Additionally, our data showed that pIVA during IRT was less sensitive to preload.

\section{Limitations}

Relatively laborious offline measurements are required to obtain the acceleration curves from TDI data. Also, a very high frame rate may require narrowing of the TDI sector angle in some patient studies. In the present study, we set a TDI sector angle to encompass the LV cavity and wall. TDI measurements were completely synchronised in both the septum and the LV lateral wall, but a decrease of frame rate was unavoidable. By narrowing the TDI sector size, high frame rates ( $>150$ to 200 frames/s) can be obtained. In reality, this is not clinically applicable for diagnosis.

\section{Conclusions}

pIVA is considered to be a sensitive and preload independent marker for evaluating LV function. pIVA observed in IVS during IRT correlates well with negative $\mathrm{dP} / \mathrm{dt}$ and pIVA observed in the LV wall during ICT correlates well with positive $\mathrm{dP} / \mathrm{dt}$.

\section{Authors' affiliations}

I Hashimoto, X-K Li, A H Bhat, D J Sahn, Clinical Care Center for Congenital Heart Disease, Oregon Health \& Science University, Portland, Oregon, USA

M Jones, National Heart, Lung, and Blood Institute, Bethesda, Maryland, USA

Competing interests: David J Sahn is an occasional consultant for GE Medical Systems. We do not believe this has had any effect on the conduct of this study. None of the other authors have any conflict of interest.

\section{REFERENCES}

1 Oki T, Mishiro Y, Yamada H, et al. Detection of left ventricular regional relaxation abnormalities and asynchrony in patients with hypertrophic cardiomyopathy with the use of tissue Doppler imaging. Am Heart J 2000; 139:497-502.

2 Edvardsen $\mathrm{T}$, Aakhus S, Endresen $\mathrm{K}$, et al. Acute regional myocardial ischemia identified by 2 -dimensional multiregion tissue Doppler imaging technique. J Am Soc Echocardiogr 2000;13:986-94.

3 Nagueh SF, Kopelen HA, Lim DS, et al. Tissue Doppler imaging consistently detects myocardial contraction and relaxation abnormalities, irrespective of cardiac hypertrophy, in a transgenic rabbit model of human hypertrophic cardiomyopathy. Circulation 2000;102:1346-50.

4 Abraham TP, Belohlavek M, Thomson HL, et al. Time to onset of regional relaxation: feasibility, variability and utility of a novel index of regional myocardial function by strain rate imaging. J Am Coll Cardiol 2002;39:1531-7.

5 Urheim S, Edvardsen T, Torp H, et al. Myocardial strain by Doppler echocardiography: validation of a new method to quantify regional myocardial function. Circulation 2000;102:1158-64.

6 Greenberg NL, Firstenberg MS, Castro PL, et al. Doppler-derived myocardial systolic strain rate is a strong index of left ventricular contractility. Circulation 2002; 105:99-105.

7 Eder V, Marchal C, Tranquart F, et al. Localization of the ventricular preexcitation site in Wolff-Parkinson-White syndrome with Doppler tissue imaging. J Am Soc Echocardiogr 2000;13:995-1001.

8 Yin LX, Li CM, Fu QG, et al. Ventricular excitation maps using tissue Doppler acceleration imaging: potential clinical application. J Am Coll Cardiol 1999;33:782-7.

9 Vogel M, Schmidt MR, Kristiansen SB, et al. Validation of myocardial acceleration during isovolumic contraction as a novel noninvasive index of right ventricular contractility: comparison with ventricular pressure-volume relations in an animal model. Circulation 2002;105:1693-9.

10 Hashimoto I, Ichida F, Miura M, et al. Automatic border detection identifies subclinical anthracycline cardiotoxicity in children with malignancy. Circulation 1999:99:2367-70.

11 Oki T, Tabata T, Yamada H, et al. Left ventricular diastolic properties of hypertensive patients measured by pulsed tissue Doppler imaging. J Am Soc Echocardiogr 1998;11:1106-12.

12 Garcia MJ, Rodriguez L, Ares M, et al. Differentiation of constrictive pericarditis from restrictive cardiomyopathy: assessment of left ventricular diastolic velocities in longitudinal axis by Doppler tissue imaging. J Am Coll Cardiol 1996;27:108-14

13 Jamal F, Strotmann J, Weidemann F, et al. Noninvasive quantification of the contractile reserve of stunned myocardium by ultrasonic strain rate and strain. Circulation 2001; 104:1059-65.

14 Saito A, Shiono $M$, Orime $Y$, et al. Effects of left ventricular assist device on cardiac function: experimental study of relationship between pump flow and left ventricular diastolic function. Artif Organs $2001 ; 25: 728-32$

15 Miyashita T, Okano Y, Takaki H, et al. Relation between exercise capacity and left ventricular systolic versus diastolic function during exercise in patients after myocardial infarction. Coron Artery Dis 2001;12:217-25.

16 Cerqueira MD, Weissman NJ, Dilsizian V, et al. Standardized myocardial segmentation and nomenclature for tomographic imaging of the heart: a statement for healthcare professionals from the cardiac imaging committee of the council on clinical cardiology of the American Heart Association. Circulation 2002; 105:539-42.

17 Harada K, Orino T, Yasuoka K, et al. Tissue Doppler imaging of left and right ventricles in normal children. Tohoku J Exp Med 2000;191:21-9.

18 Jamal F, Derumeaux G, Douillet R, et al. [Analysis and quantification of longitudinal contraction of the left ventricle in myocardial infarction: value of Doppler myocardial tissue imaging]. Arch Mal Coeur Vaiss 1999;92:315-22.

19 Kass DA, Maughan WL, Guo ZM, et al. Comparative influence of load versus inotropic states on indexes of ventricular contractility: experimental and theoretical analysis based on pressure-volume relationships. Circulation 1987;76:1422-36. 